\title{
Solvent Structure and Dynamics of the Lithium Ion in Organic Carbonate-Based Electrolytes: A Time De- pendent Infrared Spectroscopy Study
}

Kristen D. Fulfer and Daniel G. Kuroda

Department of Chemistry, Louisiana State University, Baton Rouge, LA 70803

\section{SUPPORTING INFORMATION}

\section{MODEL OF THE CARBONYL STRETCH MODES FOR A TETRAHEDRAL SOLVATION COMPLEX}

For a tetrahedrally solvated $\mathrm{Li}^{+}$, as shown in SI Figure $\mathrm{S} 1$, the frequency of each carbonyl stretch can be defined as $\omega_{10}+\delta \omega^{i}$, where $\omega_{10}$ is the frequency of the carbonyl stretch and $\delta \omega^{i}$ describes the change in frequency due to changes in the $\mathrm{Li}^{+}-$carbonyl distance. The coupling between the carbonyls is then defined by $\beta$. Then, the interactions between the carbonyl groups and the $\mathrm{Li}^{+}$can be described by the following Hamiltonian matrix:

$$
\left[\begin{array}{cccc}
\omega_{10}+\delta \omega^{1} & \beta^{12} & \beta^{13} & \beta^{14} \\
\beta^{21} & \omega_{10}+\delta \omega^{2} & \beta^{23} & \beta^{24} \\
\beta^{31} & \beta^{32} & \omega_{10}+\delta \omega^{3} & \beta^{34} \\
\beta^{41} & \beta^{42} & \beta^{43} & \omega_{10}+\delta \omega^{4}
\end{array}\right]
$$

If we assume that all the $\delta \omega$ are equal and that all the $\beta$ are equal in the solvation complex, then, the above Hamiltonian matrix becomes:

$$
\left[\begin{array}{cccc}
\omega_{10}+\delta \omega & \beta & \beta & \beta \\
\beta & \omega_{10}+\delta \omega & \beta & \beta \\
\beta & \beta & \omega_{10}+\delta \omega & \beta \\
\beta & \beta & \beta & \omega_{10}+\delta \omega
\end{array}\right]
$$

Diagonalizing the above matrix gives:

$$
\operatorname{Diag}\left[\begin{array}{cccc}
\omega_{10}+\delta \omega & \beta & \beta & \beta \\
\beta & \omega_{10}+\delta \omega & \beta & \beta \\
\beta & \beta & \omega_{10}+\delta \omega & \beta \\
\beta & \beta & \beta & \omega_{10}+\delta \omega
\end{array}\right]=\left[\begin{array}{cccc}
\omega_{10}+\delta \omega-\beta & 0 & 0 & 0 \\
0 & \omega_{10}+\delta \omega-\beta & 0 & 0 \\
0 & 0 & \omega_{10}+\delta \omega-\beta & 0 \\
0 & 0 & 0 & \omega_{10}+\delta \omega+3 \beta
\end{array}\right]
$$

Which results in three carbonyl stretches which have a frequency of $\omega_{10}+\delta \omega-\beta$, these are the asymmetric stretch modes, and one carbonyl stretch which has a frequency of $\omega_{10}+\delta \omega+3 \beta$, this is the symmetric stretch mode.

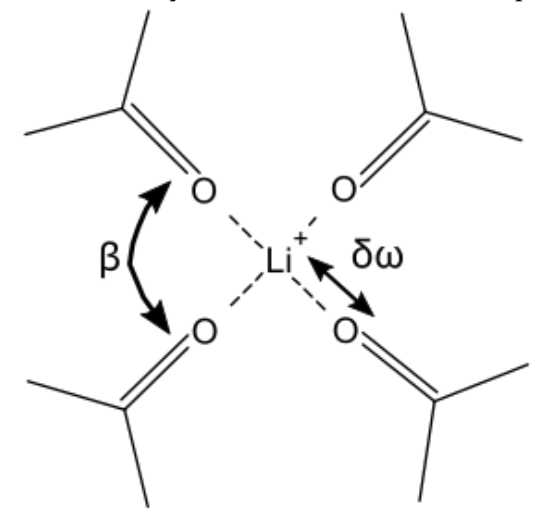

FIGURE S 1. Li ${ }^{+}$tetrahedrally solvated (though drawn planar) by four carbonyl groups. 
TABLE S 1. Fitting parameters for concentration dependent FTIR data for LiPF $_{6}$ in BC and DMC. All frequencies and widths are reported in $\mathrm{cm}^{-1}$. The most concentrated $B C$ solution had an additional low frequency peak in the carbonyl stretch region. However, the most concentrated BC solution also had a very high absorbance meaning the lineshape fitting may not be reliable.

\begin{tabular}{|c|c|c|c|c|c|c|c|c|c|}
\hline \multirow[b]{2}{*}{ Solvent } & \multirow[b]{2}{*}{$\mathbf{x}\left(\mathbf{L i}^{+}\right)$} & \multicolumn{4}{|c|}{ Free Peak } & \multicolumn{4}{|c|}{ "Li+ - coordinated Peak } \\
\hline & & Freq. & FWHM & $\begin{array}{l}\text { Gaussian } \\
\text { Width }\end{array}$ & $\begin{array}{l}\text { Lorentz. } \\
\text { Width }\end{array}$ & Freq. & FWHM & $\begin{array}{l}\text { Gaussian } \\
\text { Width }\end{array}$ & $\begin{array}{l}\text { Lorentz. } \\
\text { Width }\end{array}$ \\
\hline \multirow{7}{*}{$\mathrm{BC}$} & o & 1800.06 & $47 \cdot 7$ & $33 \cdot 3$ & 14.8 & & & & \\
\hline & 0.05 & 1802 & 47 & 32 & 16.9 & 1776 & 49 & 31 & 21 \\
\hline & 0.10 & 1804.08 & 40.1 & 25.6 & 16.8 & $1774 \cdot 3$ & 45.2 & 25.6 & 16.8 \\
\hline & 0.15 & 1805.6 & 35.1 & 21.7 & 15.8 & $1773 \cdot 7$ & 43.6 & 29.1 & 16.0 \\
\hline & 0.21 & 1806.6 & 30.2 & $25 \cdot 7$ & o & $1773 \cdot 5$ & 40.1 & $17 \cdot 0$ & 29.6 \\
\hline & 0.27 & $1805 \cdot 7$ & 29 & 24 & o & $1773 \cdot 7$ & 37.2 & 12.5 & 31.0 \\
\hline & & & & & & 1735 & 45 & 38 & o \\
\hline \multirow{6}{*}{ DMC } & o & 1756.72 & 20.3 & 6.6 & 17.2 & & & & \\
\hline & 0.04 & 1756.90 & 22.0 & 9.9 & $15 \cdot 5$ & 1722.8 & 20 & o & 20 \\
\hline & 0.09 & 1757.17 & 22.9 & 13.5 & 11.4 & 1723.86 & 22.3 & o & 22.3 \\
\hline & 0.13 & 1756.98 & 23.5 & 12.6 & 13.8 & $1723 \cdot 51$ & 22.1 & o & 22.1 \\
\hline & 0.19 & 1756.26 & $25 \cdot 4$ & 18.9 & $5 \cdot 6$ & $1724 \cdot 34$ & 22.8 & o & 22.8 \\
\hline & 0.24 & 1754.1 & 31.1 & 23.5 & 6.0 & 1724.63 & 23.8 & o & 23.8 \\
\hline
\end{tabular}

TABLE S 2. Exponential modeling results for the FTIR fractional area of the coordinated carbonyl stretch band for each electrolyte as a function of $\mathrm{X}\left(\mathrm{Li}^{+}\right)$.

\begin{tabular}{l|c|c}
\hline \hline & yo & C \\
\hline \hline BC & $1.3 \pm 0.2$ & $0.21 \pm 0.06$ \\
\hline DMC & $1.1 \pm 0.2$ & $0.18 \pm 0.05$ \\
\hline \hline
\end{tabular}

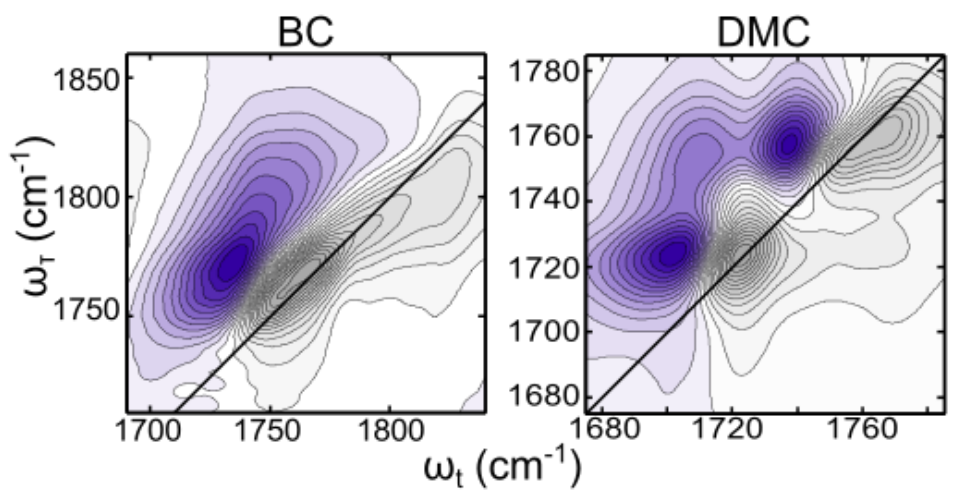

FIGURE S 2. 2DIR spectra of LiPF6 in BC (left) and DMC (right) at $\mathrm{X}\left(\mathrm{Li}^{+}\right)=0.09$ for waiting time $\mathrm{T}_{\mathrm{w}}=0$ ps with $\mathrm{k}_{1}$ and $\mathrm{k}_{2}$ at $45^{\circ}$. 
SCHEME S 1. Geometries used in the DFT frequency calculations for the cyclic organic carbonates (CC).

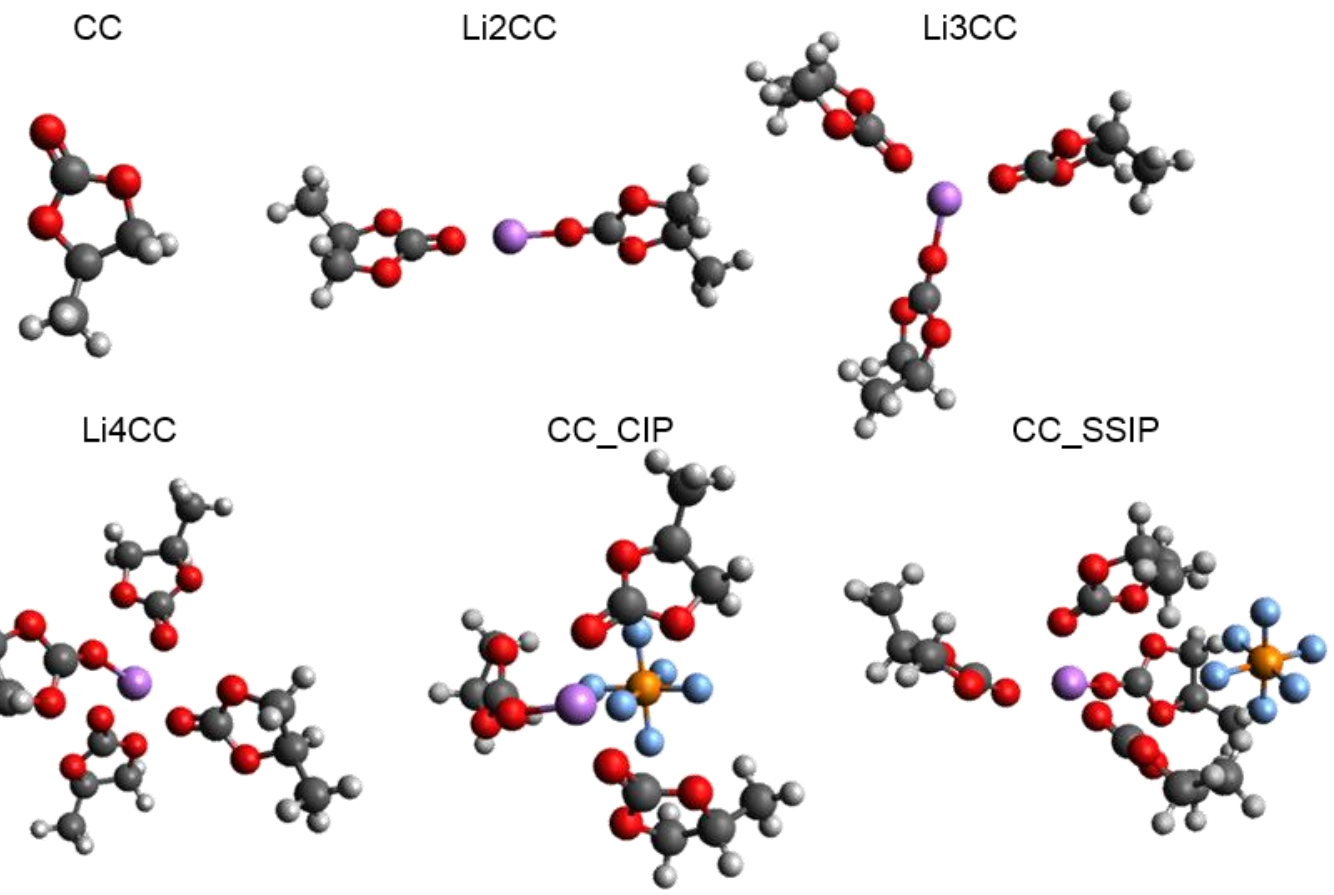

TABLE S 3. DFT results for the carbonyl stretch mode frequencies for the cyclic carbonate (CC) solvation complexes calculated in gas phase. All frequencies are reported in $\mathrm{cm}^{-1}$; intensities are reported in $\mathbf{k m} / \mathbf{m o l}$.

\begin{tabular}{|c|c|c|c|c|c|c|}
\hline \multirow{2}{*}{ Species } & \multicolumn{2}{|c|}{ Free ${ }^{* *}$ calculated separately } & \multicolumn{2}{|c|}{ Symmetric } & \multicolumn{2}{|c|}{ Asymmetric } \\
\hline & Freq. & Intensity & Freq. & Intensity & Freq. & Intensity \\
\hline $\mathrm{Li}^{+}{ }_{2} \mathrm{CC}$ & 1891.61 & $725 \cdot 57$ & 1819.01 & 0.05 & 1791.19 & 2411.13 \\
\hline $\mathrm{Li}^{+}{ }_{3} \mathrm{CC}$ & 1891.61 & $725 \cdot 57$ & 1863.14 & 0.44 & $\begin{array}{l}1821.19 \\
1821.02\end{array}$ & $\begin{array}{l}1703.06 \\
1767.44\end{array}$ \\
\hline $\mathrm{Li}^{+}{ }_{4} \mathrm{CC}$ & 1891.61 & $725 \cdot 57$ & 1875.01 & 0.34 & $\begin{array}{l}1834.89 \\
1834.45 \\
1831.87\end{array}$ & $\begin{array}{c}1300.62 \\
1302.82 \\
1516.49\end{array}$ \\
\hline $\mathrm{CIP}\left(\mathrm{Li}^{+} \mathrm{PF}_{6}^{-}{ }_{3} \mathrm{CC}\right)$ & 1891.61 & $725 \cdot 57$ & 1846.42 & 752.86 & $\begin{array}{l}1815.87 \\
1810.77\end{array}$ & $\begin{array}{l}936.53 \\
663.04\end{array}$ \\
\hline $\operatorname{SSIP}\left(\mathrm{Li}^{+} \mathrm{PF}_{6}{ }_{4}{ }_{4} \mathrm{CC}\right)$ & 1891.61 & $725 \cdot 57$ & 1864.87 & 288.28 & $\begin{array}{l}1834.99 \\
1816.26 \\
1803.24\end{array}$ & $\begin{array}{l}2127.31 \\
504.90 \\
543.98\end{array}$ \\
\hline
\end{tabular}


TABLE S 3. DFT results for the carbonyl stretch mode frequencies for the linear carbonate (DMC) solvation complexes calculated in gas phase. All frequencies are reported in $\mathrm{cm}^{-1}$; intensities are reported in $\mathbf{k m} / \mathbf{m o l}$.

\begin{tabular}{|c|c|c|c|c|c|c|}
\hline \multirow{2}{*}{ Species } & \multicolumn{2}{|c|}{ Free ${ }^{* *}$ calculated separately } & \multicolumn{2}{|c|}{ Symmetric } & \multicolumn{2}{|c|}{ Asymmetric } \\
\hline & Frequency & Intensity & Frequency & Intensity & Frequency & Intensity \\
\hline $\mathrm{Li}^{+} 2 \mathrm{LC}$ & 1823.52 & 529.65 & 1737.45 & 0.00 & 1713.8 & 1566.55 \\
\hline $\mathrm{Li}^{+}{ }_{3} \mathrm{LC}$ & $1823 \cdot 52$ & 529.65 & 1780.00 & 37.24 & $\begin{array}{l}1744.55 \\
1742.07\end{array}$ & $\begin{array}{l}1257.91 \\
967.90\end{array}$ \\
\hline $\mathrm{Li}^{+}{ }_{4} \mathrm{LC}$ & 1823.52 & 529.65 & 1811.01 & 242.13 & $\begin{array}{c}1769.08 \\
1767.28 \\
1766.64\end{array}$ & $\begin{array}{l}508.23 \\
555.79 \\
1411.07\end{array}$ \\
\hline $\mathrm{CIP}\left(\mathrm{Li}^{+} \mathrm{PF}_{6}{ }^{-} \mathrm{LC}^{2}\right)$ & $1823 \cdot 52$ & 529.65 & 1813.98 & 56.33 & $\begin{array}{l}1783.03 \\
1767.2\end{array}$ & $\begin{array}{l}1306.76 \\
818.70\end{array}$ \\
\hline $\operatorname{SSIP}\left(\mathrm{Li}^{+} \mathrm{PF}_{6}{ }_{4} \mathrm{LC}\right)$ & $1823 \cdot 52$ & 529.65 & 1811.06 & $125 \cdot 31$ & $\begin{array}{l}1773.45 \\
1750.60 \\
1745.76\end{array}$ & $\begin{array}{l}1449.40 \\
747.844 \\
369.989\end{array}$ \\
\hline
\end{tabular}

SCHEME S 2. Geometries in the DFT frequency calculations for the linear organic carbonates (LC).

LC

Li2LC

Li3LC
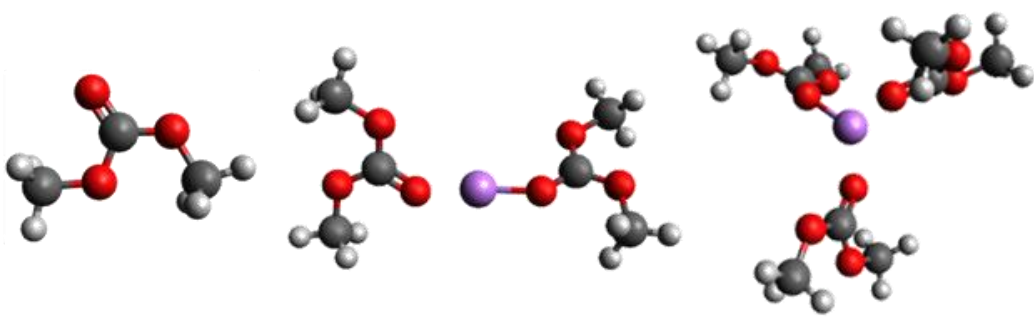

Li4LC
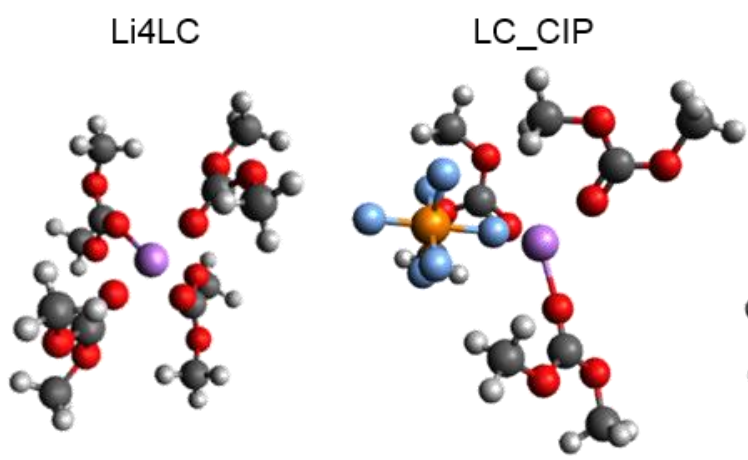

LC_SSIP

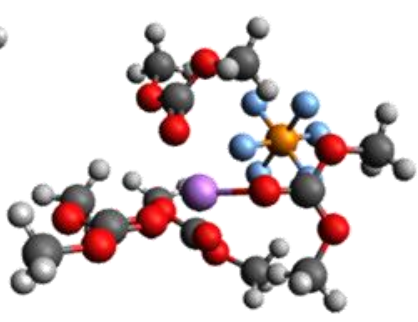



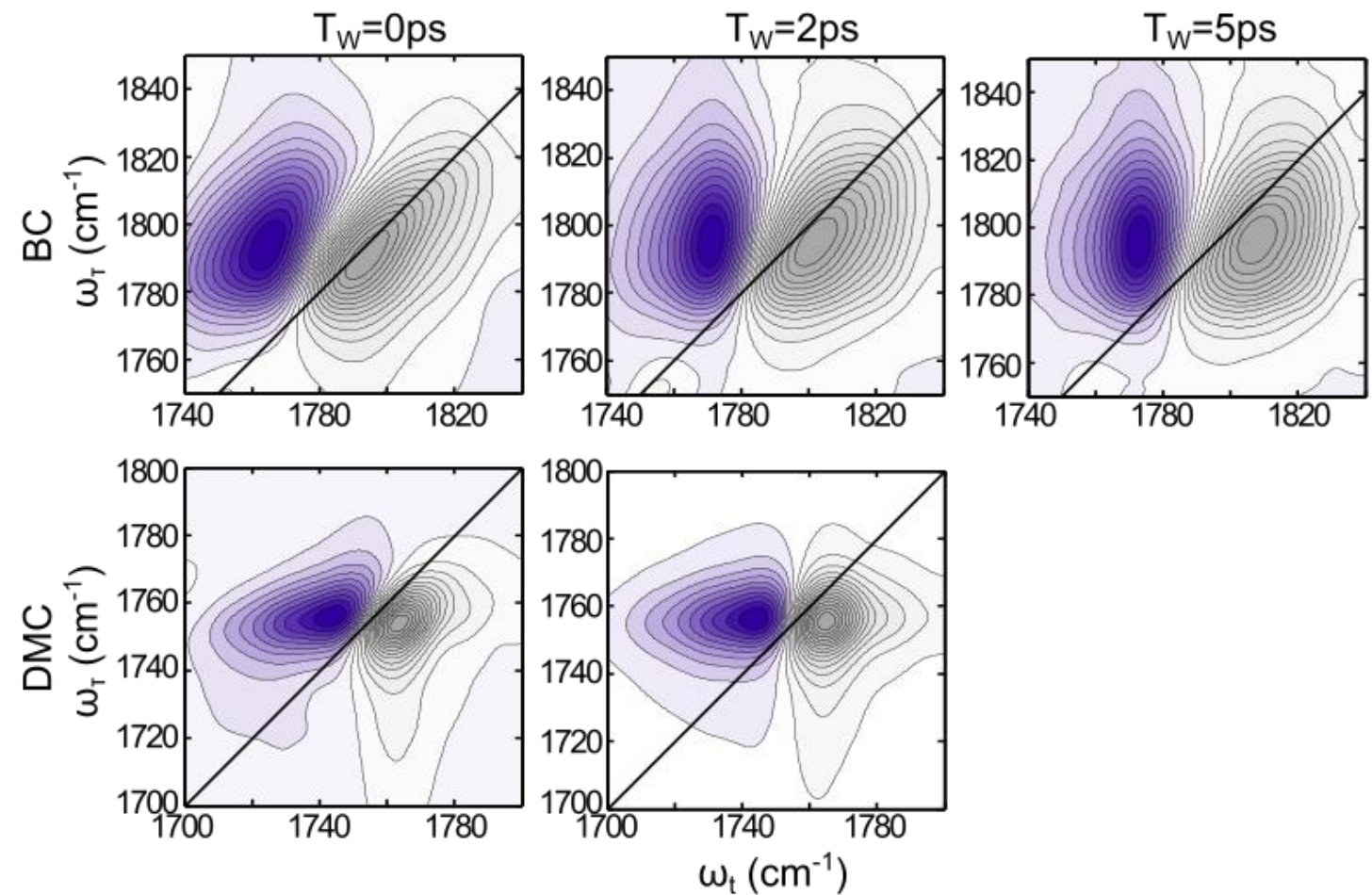

FIGURE S 3. 2DIR spectra of neat organic carbonates: BC (top row) and DMC (bottom row) for waiting times $\mathrm{T}_{\mathrm{w}}=0$ ps (left column), $\mathrm{T}_{\mathrm{w}}=2 \mathrm{ps}$ (middle column), and $\mathrm{T}_{\mathrm{w}}=5$ (right column). Note that the DMC data was not collected for $\mathrm{T}_{\mathrm{w}}=5.0 \mathrm{ps}$. 